\title{
Estonian Vegetation Database
}

\author{
Jaanus Paal
}

\begin{abstract}
The main aim of the Estonian Vegetation Database (GIVD ID EU-EE-001) is to provide, as complete as possible, a synopsis of Estonian plant community types, to enable analysis of their species diversity on synusiae/microcoenoses, community and regional scale, to assess the management impact on vegetation and to study successional processes in plant communities. For example, in official Estonian forest site type classification interprets several types rather widely and habitats of some dispersed types (floodplain, hillock and klint/escarpment forests) having great value for biodiversity preservation have been overlooked. In 2011 the inventory of Estonian mire habitats (15,326 in total) was completed, partly including vegetation data, but the latter have not been analysed yet. The most serious shortcoming of the Estonian vegetation database is until now the disunion of grassland data.
\end{abstract}

Keywords: forest site type; grassland; microcoenoses; mire; synusia.

GIVD Database ID: EU-EE-001

Last update: 2011-07-06

\section{Estonian Vegetation Database}

Scope: Numbers of relevés: Watercourses $n=750$; Alvar forests $n=130$; boreo-nemoral forests $n=250$; swamp forests; $n=150$; paludified and mire forests $n=100$; mires $n=50$.

Status: ongoing capture

Period: $1980-2010$

Database manager(s): Jaanus Paal (jaanus.paal@ut.ee)

Owner: University of Tartu

Web address: http://www.botany.ut.ee/

Availability: according to a specific agreement

Database format(s): Excel

Publication: [NA]

Plot type(s): normal plots

Non-overlapping plots: 1,430

Total plot observations: 1,430

Period: 1980-2010

Countries: EE: $98.0 \%$; SE: $2.0 \%$

Forest: [NA] — Non-forest: [NA]

Guilds: all vascular plants: $100 \%$; bryophytes (terricolous or aquatic): $100 \%$; lichens (terricolous or aquatic): $5 \%$; algae (terricolous or aquatic):

$40 \%$; non-terricolous taxa (epiphytic, saxicolous, lignicolous): $5 \%$

Environmental data: surface cover other than plants (open soil, litter, bare rock etc.): $50 \%$; soil pH: $50 \%$; land use categories: $50 \%$

Performance measure(s): cover: $100 \%$; measurements like diameter or height of trees: $100 \%$

Geographic localisation: GPS coordinates (precision $25 \mathrm{~m}$ or less): 50\%; small grid (not coarser than $10 \mathrm{~km}$ ): $50 \%$

Sampling periods: 1980-1989: 40.0\%; 2000-2009: 45.0\%; 2010-2019: 5.0\%; unknown: 10.0\%

Information as of 2012-07-12; further details and future updates available from http://www.givd.info/ID/EU-EE-001

Jaanus Paal (jaanus.paal@ut.ee)

Department of Botany, University of Tartu, Lai 40, 51005 Tartu, ESTONIA 